\title{
Regular braneworlds with nonlinear bulk-fluids
}

\author{
Ignatios Antoniadis $^{1,2, a}$, Spiros Cotsakis ${ }^{3,4, b}$, Ifigeneia Klaoudatou ${ }^{4, c}$ \\ ${ }^{1}$ Laboratoire de Physique Théorique et Hautes Energies-LPTHE, Sorbonne Université, CNRS 4 Place Jussieu, 75005 Paris, France \\ ${ }^{2}$ Institute for Theoretical Physics, KU Leuven, Celestijnenlaan 200D, 3001 Leuven, Belgium \\ ${ }^{3}$ Institute of Gravitation and Cosmology, RUDN University, ul. Miklukho-Maklaya 6, Moscow 117198, Russia \\ ${ }^{4}$ Research Laboratory of Geometry, Dynamical Systems and Cosmology, University of the Aegean, Karlovassi, 83200 Samos, Greece
}

Received: 23 July 2021 / Accepted: 16 August 2021 / Published online: 26 August 2021

(C) The Author(s) 2021

\begin{abstract}
We construct a regular five-dimensional braneworld with localised gravity on a flat 3-brane. The matter content in the bulk is parametrised by an analog of a nonlinear fluid with equation of state $p=\gamma \rho^{\lambda}$ between the 'pressure' $p$ and the 'density' $\rho$ dependent on the 5 th dimension. For $\gamma$ negative and $\lambda>1$, the null energy condition is satisfied and the geometry is free of singularities within finite distance from the brane, while the induced four-dimensional Planck mass is finite.
\end{abstract}

\section{Contents}

1 Introduction ................... 1

2 Evolution equations and consistency . . . . . . . 2

2.1 Field equations . . . . . . . . . . . . 2

2.2 Energy conditions . . . . . . . . . . . . 2

3 Representation of solutions depending on $\lambda \ldots \ldots 4$

3.1 Some properties of the $\lambda$-solutions . . . . . 4

3.2 Impossibility of viable solutions for $\lambda<1 \ldots 5$

4 The $\lambda>1$ solutions . . . . . . . . . 6

4.1 Construction. . . . . . . . . . . 6

4.2 Regularity . . . . . . . . . . . . 7

5 Matching and localization: a special case . . . . . . 8

5.1 Derivatives ............... . . 9

5.2 Matching at the inflection point . . . . . . . 9 9

5.3 Localization . . . . . . . . . . . . . . 10

6 Matching: the general case . . . . . . . . . . . 11

7 Localization . . . . . . . . . . . . . . 13

8 Conclusions ............... . . 14

References . . . . . . . . . . . . . . . . . . . . 14

a e-mail: antoniad@lpthe.jussieu.fr

b e-mail: skot@aegean.gr

ce-mail: iklaoud@aegean.gr (corresponding author)

\section{Introduction}

In previous works [1-5], we have made a detailed investigation of the singularity structure of a five-dimensional (5d) brane-world, consisting on a 3-brane embedded in five dimensions with bulk matter content parametrised by an ana$\log$ of a perfect fluid with equation of state $p=\gamma \rho$, between the 'pressure' $p$ and the 'density' $\rho$ dependent on the 5th coordinate $Y$, playing the role of time. This work extended previous studies of brane-worlds with bulk scalar fields [68]. The parameter $\gamma$ should be greater than -1 , in order to satisfy the null energy condition (guaranteeing the absence of ghosts in the bulk). It turns out that for a flat brane, there is always a singularity that appears in the bulk within finite distance from the brane position $[2,4]$. The singularity can be avoided by considering half of the space, starting from the brane position to the side which is singularity free and using appropriate junction (matching) conditions [4]. The resulting construction however does not localise gravity on the brane and the effective $4 \mathrm{~d}$ Planck mass is divergent. These results essentially continue to hold when the brane is curved and maximally symmetric. In this case, the null energy condition restricts $\gamma$ to be in the interval $(-1,-1 / 2)$ and the brane is negatively curved (anti-de Sitter), but still does not localise gravity [5].

In this work, we extend our analysis by considering a flat brane and replacing the bulk content with an analog of a non-linear fluid, satisfying an equation of state $p=\gamma \rho^{\lambda}$. Formally similar equations of state have been used in various cosmological contexts such as in studies of escaping big-rip singularities during late time asymptotics [9-11], in obtaining inflationary models with special properties [12], as unified models of dark energy and dark matter $[13,14]$, as well as in studies of singularities [15-17].

We show that for $\gamma$ negative and $\lambda>1$, the null energy condition is satisfied and there is no singularity within finite distance from the brane. Moreover, we are able to find explicit 
general solutions in terms of hypergeometric functions that further simplify considerably for particular values of $\lambda=1+$ $1 /(2 k)$ with $k$ a positive integer. It is important to emphasise that these solutions are regular independently of the presence of the brane. When the latter is taken into account a cutting and matching procedure is needed for half of the space, as explained above, which can be done in a way that the integral for the induced $4 \mathrm{~d}$ effective Planck mass is finite, leading to localised gravity on the brane for any value of the exponent $\lambda>1$. In the perfect fluid limit $\lambda \rightarrow 1$, the 4 d Planck mass diverges and in agreement with the previous analysis.

The structure of this paper is as follows. In the next section, we write down the system of dynamical equations holding in the bulk ( $Y$-coordinate), together with the nonlinear equation of state assumed for the bulk fluid. This equation introduces a new parameter $\lambda$ into the problem that plays a central role in the following. We also derive the precise restrictions imposed by the validity of the energy conditions. In Sect. 3, we introduce the desirable properties of the solutions and show how these depend crucially on $\lambda$. In the remaining Sects. 4-7, we study in detail the properties of solutions which have both the sought for properties, namely, they are free of finite $Y$-distance singularities and have a finite Planck mass. We construct these solutions and also show how their matching is done to produce the said behaviour. We conclude our work in the last section.

\section{Evolution equations and consistency}

In this section, we setup the field equations of a brane embedded in a 5-bulk space which is filled with an imperfect fluid with a nonlinear equation of state, and write down the restrictions imposed by the energy conditions on the bulk fluid quantities by the 5-dimensional geometry.

\subsection{Field equations}

Our braneworld model consists of a 3-brane embedded in a five-dimensional bulk space with a metric of the form,

$g_{5}=a^{2}(Y) g_{4}+d Y^{2}$,

where $a(Y)$ is the warp factor with $a(Y)>0$, which we simply denote by $a$, while $g_{4}$ is the four-dimensional flat metric, i.e.,

$g_{4}=-d t^{2}+d x_{1}^{2}+d x_{2}^{2}+d x_{3}^{2}$.

Our sign conventions are defined as follows. We take capital Latin indices $A, B, \cdots=1,2,3,4,5$, Greek indices $\alpha, \beta, \cdots=1,2,3,4$, with $t$ being the timelike coordinate, and $\left(x_{1}, x_{2}, x_{3}, Y\right)$ the remaining spacelike ones. The 5dimensional Riemann tensor is defined by the formula,

$$
R_{B C D}^{A}=\partial_{C} \Gamma_{B D}^{A}-\partial_{D} \Gamma_{B C}^{A}+\Gamma_{B D}^{M} \Gamma_{M C}^{A}-\Gamma_{B C}^{M} \Gamma_{M D}^{A}
$$

the Ricci tensor is the contraction,

$R_{A B}=R_{A C B}^{C}$,

and the five-dimensional Einstein equations on the bulk space are given by,

$G_{A B}=R_{A B}-\frac{1}{2} g_{A B} R=\kappa_{5}^{2} T_{A B}$.

We assume a bulk fluid having an energy-momentum tensor of the form,

$T_{A B}=(\rho+p) u_{A} u_{B}-p g_{A B}$,

with a nonlinear equation of state,

$p=\gamma \rho^{\lambda}$,

where the 'pressure' $p$ and the 'density' $\rho$ are functions only of the fifth coordinate $Y$ and $\gamma, \lambda$ are constants. The fluid velocity vector field is $u_{A}=(0,0,0,0,1)$, that is $u_{A}=$ $\partial / \partial Y$, parallel to the $Y$-dimension.

The Einstein equations and the equation of energymomentum conservation, $\nabla_{A} T^{A B}=0$, then become,

$$
\begin{aligned}
& \frac{a^{\prime 2}}{a^{2}}=\frac{\kappa_{5}^{2}}{6} \rho, \\
& \frac{a^{\prime \prime}}{a}=-\frac{\kappa_{5}^{2}}{6}\left(2 \gamma \rho^{\lambda}+\rho\right), \\
& \rho^{\prime}+4\left(\gamma \rho^{\lambda}+\rho\right) \frac{a^{\prime}}{a}=0 .
\end{aligned}
$$

where the prime $\left(^{\prime}\right)$ denotes differentiation with respect to $Y$. Solving the system of equations Eqs. (2.8), (2.9) and (2.10), entails constructing a scheme of solutions based on the ranges of the parameters $\gamma$ and $\lambda$. Our goal is to determine the ranges of parameters that lead to solutions that satisfy physical conditions such as the energy conditions. To this end, we first study the energy conditions and the restrictions that they imply on the density and pressure of the fluid, we then express them in terms of the ranges of the parameters and finally we include them in our solutions. In this way, we integrate from the beginning the energy conditions in our final forms of solutions.

\subsection{Energy conditions}

We now move on to study the restrictions imposed by the strong, weak and null energy conditions on the bulk fluid (2.6). These translate to conditions to be satisfied by $p$ and $\rho$, and constitute a priori restrictions on our solutions in the form of physical conditions.

In our formulation of the field equations, the metric (2.1) and the bulk fluid appear as static with respect to the time 
coordinate $t$, because the evolution is taken with respect to the fifth spatial coordinate $Y$. We have however the unitarity constraint imposed on the model with regard to the $t$-evolution under which our $5 \mathrm{~d}$ fluid is anisotropic in the $Y$-direction. Here, we take into account this positive energy constraint on the brane-bulk system following the analysis of [4].

To work out the possible restrictions imposed on the system by the requirement of consistency of the evolution in the $\partial / \partial t$ direction with that along the $\partial / \partial Y$ vector field, we shall use the energy conditions. To proceed, we reinterpret our fluid analogue as a real anisotropic fluid having the following energy momentum tensor,

$T_{A B}=\left(\rho^{0}+p^{0}\right) u_{A}^{0} u_{B}^{0}-p^{0} g_{\alpha \beta} \delta_{A}^{\alpha} \delta_{B}^{\beta}-p_{Y} g_{55} \delta_{A}^{5} \delta_{B}^{5}$,

where $u_{A}^{0}=(a(Y), 0,0,0,0), A, B=1,2,3,4,5$ and $\alpha, \beta=1,2,3,4$. When we combine (2.6) with (2.11), we get the following set of relations,

$p_{Y}=-\rho$

$\rho^{0}=-p$

$p^{0}=p$.

The last two relations imply that

$p^{0}=-\rho^{0}$,

which means that this type of matter satisfies a cosmological constant-like equation of state. Substituting (2.12)-(2.15) in (2.11), we find that

$T_{A B}=-p g_{\alpha \beta} \delta_{A}^{\alpha} \delta_{B}^{\beta}+\rho g_{55} \delta_{A}^{5} \delta_{B}^{5}$.

We are now ready to form the energy conditions for our type of matter. We begin with the weak energy condition according to which, every future-directed timelike vector $v^{A}$ should satisfy

$T_{A B} v^{A} v^{B} \geq 0$.

This condition implies that the energy density should be non negative for all forms of physical matter [18]. Here we find (see [4] and [5] for detailed proofs) that it translates to

$p \geq 0, \quad$ or, equivalently, $\quad \gamma \geq 0$

and

$p+\rho \geq 0$.

Inputting the equation of state $p=\gamma \rho^{\lambda}$ in Eq. (2.19) we find

$\gamma \rho^{\lambda}+\rho=\rho^{\lambda}\left(\gamma+\rho^{1-\lambda}\right) \geq 0$

and since $\rho \geq 0$ from Eq. (2.8), we simply get

$\gamma+\rho^{1-\lambda} \geq 0$.
The strong energy condition, on the other hand, demands that

$$
\left(T_{A B}-\frac{1}{3} T g_{A B}\right) v^{A} v^{B} \geq 0,
$$

for every future-directed unit timelike vector $v^{A}$. In our case, this condition leads to the following restrictions for $p$ and $\rho$,

$-p+\rho \geq 0$,

and

$p+\rho \geq 0$.

Again, Eq. (2.23) can be written equivalently

$-\gamma \rho^{\lambda}+\rho=\rho^{\lambda}\left(-\gamma+\rho^{1-\lambda}\right) \geq 0$

and using the fact that $\rho \geq 0$, we get

$-\gamma+\rho^{1-\lambda} \geq 0$.

Similarly, from Eq. (2.24) we find

$\gamma+\rho^{1-\lambda} \geq 0$.

Finally, according to the null energy condition, every future-directed null vector $k^{A}$ should satisfy [19]

$T_{A B} k^{A} k^{B} \geq 0$.

Here we find that it translates to

$p+\rho \geq 0$,

which implies, as we have already seen, that

$\gamma+\rho^{1-\lambda} \geq 0$.

This equation together with the Friedman equation (2.8) give the following bound for the scale factor as a function of the $Y$ coordinate,

$a(Y) \geq e^{\sqrt{\rho_{\min }} Y}$,

where $\rho_{\min }=(-\gamma)^{1 /(1-\lambda)}$.

To compare between the effects of a linear and non-linear equation of state on the restrictions set by the energy conditions, we find it useful to mention here that for the case of a linear equation of state $(\lambda=1)$, the weak energy condition requires $p \geq 0$ and $\gamma>0$, while the strong energy condition restricts $p$ and $\gamma$ to either $p<0$ and $-1 \leq \gamma<0$, or, $p>0$ and $0<\gamma \leq 1$ [4].

Summarizing the constraints set by the energy conditions for a non-linear fluid, we see that the restriction described by (2.30) is required by all energy conditions and we shall take this into account in the next section in the procedure of deriving our solutions. 


\section{Representation of solutions depending on $\lambda$}

In this section, we give general properties of the solutions of equations Eqs. (2.8), (2.9) and (2.10). The desirable properties we seek for these solutions are:

- regularity

- localization of gravity on the brane.

For regularity, solutions should not have finite- $Y$-distance singularities, whereas the localization property is effected with a finite Planck mass. We shall discuss these two properties at length in later sections.

\subsection{Some properties of the $\lambda$-solutions}

Since the continuity equation (2.10) is a separable equation, by integrating it and substituting into the Friedman equation (2.8), we can arrive at a general relation between the density and the warp factor satisfied by the system (2.8), (2.9) and (2.10).

While integrating, a logarithmic term of the form $\ln \mid \gamma+$ $\rho^{1-\lambda} \mid$ appears. We ignore the absolute value and simply put this term equal to $\ln \left(\gamma+\rho^{1-\lambda}\right)$, in compliance with the restriction (2.30) of the previous section as required by all energy conditions. We the end up with,

$\rho=\left(-\gamma+c_{1} a^{4(\lambda-1)}\right)^{1 /(1-\lambda)}$,

where

$c_{1}=\frac{\gamma+\rho_{0}^{1-\lambda}}{a_{0}^{4(\lambda-1)}}$,

with $\rho_{0}=\rho\left(Y_{0}\right), a_{0}=a\left(Y_{0}\right)$ the initial conditions. We note that (2.30) now implies that $c_{1} \geq 0$, while the strong energy condition translates to,

$-2 \gamma+c_{1} a^{4(\lambda-1)} \geq 0$.

It is important to note from Eq. (3.1) that $\rho$ becomes infinite for $\lambda>1$, and

$a^{4(\lambda-1)}=\frac{\gamma}{c_{1}}$.

To avoid this singularity, we are going to focus only on negative values of $\gamma$, which means that the weak energy condition is not going to be satisfied by our solutions. However, the null energy condition is already embodied in our solutions and the strong energy condition (3.3) is automatically satisfied for $\gamma<0$.

We continue by substituting Eq. (3.1) in Eq. (2.8), to obtain,

$\int \frac{a}{\left(c_{1}-\gamma a^{4(1-\lambda)}\right)^{1 /(2(1-\lambda))}} d a= \pm \frac{\kappa_{5}}{\sqrt{6}} \int d Y$.
Further examination of this equation reveals that solutions depend crucially on whether $\lambda$ is greater or smaller than one. All solutions can be grouped in these two large classes, and this classification is also consistent with the dynamics around finite equilibria, or at infinity, cf. [20].

For $\lambda$ greater than one, the integral on the left-hand side of Eq. (3.5) can be directly integrated out for those values of $\lambda$ that make $1 /(2(1-\lambda))$ a negative integer. This is possible when $\lambda=(n+1) / n$, with $n=2 k$ and $k$ a positive integer.

Take for example the simplest case $n=2$ which gives $\lambda=3 / 2$. Then $1 /(2(1-\lambda))=-1$ and we can directly integrate Eq. (3.5) to get the solution in the following implicit form

$\pm Y+c_{2}=\frac{\sqrt{6}}{\kappa_{5}}\left(\frac{c_{1}}{2} a^{2}-\gamma \ln a\right)$

where $c_{2}$ is an integration constant. We can now go one step further and derive the general form of solution for $\lambda=(n+$ 1) $/ n, n=2 k$ and $k$ a positive integer. Using the binomial theorem, integrating and wrapping up terms we find,

$$
\begin{aligned}
\pm Y+c_{2}= & \frac{\sqrt{6}}{\kappa_{5}}\left(\sum_{s=0}^{k-1} \frac{k !}{(k-s) ! s !} \frac{c_{1}^{k-s}}{2-2 s / k}\right. \\
& \left.\times a^{2-2 s / k}(-\gamma)^{s}+(-\gamma)^{k} \ln a\right) .
\end{aligned}
$$

For all values of $\lambda$ such that $\lambda \neq(n+1) / n$, the integral on the left hand-side of Eq. (3.5) cannot in general be calculated directly. In this case, we can express the solutions in terms of the Gaussian hypergeometric function ${ }_{2} F_{1}(\alpha, b, c ; z)$, defined by

${ }_{2} F_{1}(\alpha, b, c ; z)=\frac{\Gamma(c)}{\Gamma(\alpha) \Gamma(b)} \sum_{n=0}^{\infty} \frac{\Gamma(\alpha+n) \Gamma(b+n)}{\Gamma(c+n)} \frac{z^{n}}{n !}$

and convergent for $|z|<1$, where $\Gamma$ is the Gamma function given by

$\Gamma(z)=\int_{0}^{\infty} e^{-t} t^{z-1} d t, \quad \operatorname{Re}(z)>0$.

An integral representation of ${ }_{2} F_{1}(\alpha, b, c ; z)$ is [21]

$$
\begin{aligned}
{ }_{2} F_{1}(\alpha, b, c ; z)= & \frac{\Gamma(c)}{\Gamma(b) \Gamma(c-b)} \\
& \times \int_{0}^{1} t^{b-1}(1-t)^{c-b-1}(1-t z)^{-\alpha} d t,
\end{aligned}
$$

provided that

$0<\operatorname{Re}(b)<\operatorname{Re}(c)$. 
The RHS of (3.10) is a one-valued analytic function $z$ within the domain

$$
|\arg (1-z)|<\pi \text {. }
$$

This converges for $|z|<1$. However, because of the definition of $z$ and the fact that on the circle of convergence there is a singular point given by Eq. (3.4), it follows that by analytic continuation we can extend the disk of convergence far beyond this limit (cf. e.g., [22], pp. 389-90).

To derive our solution in terms of a hypergeometric function, we simply set

$z=\gamma a^{4(1-\lambda)} / c_{1}$

and note that the restriction (3.12) translates into,

$|\arg (1-z)|=\left|\arg \left(\frac{c_{1}-\gamma a^{4(1-\lambda)}}{c_{1}}\right)\right|<\pi$,

which is satisfied since $-\gamma$ and $c_{1}$ are both positive real numbers. We arrive at the following form of our solutions,

$$
\begin{aligned}
\pm Y+c_{2}= & \frac{\sqrt{6}}{4(1-\lambda) \kappa_{5}} c_{1}^{1 /(2(\lambda-1))} a^{2} \int_{0}^{1} w^{1 /(2(1-\lambda))-1} \\
& \times\left(1-\frac{\gamma}{c_{1}} a^{4(1-\lambda)} w\right)^{-1 /(2(1-\lambda))} d w \\
= & \frac{\sqrt{6}}{2 \kappa_{5}} c_{1}^{1 /(2(\lambda-1))} a^{2}{ }_{2} F_{1}\left(\frac{1}{2(1-\lambda)}, \frac{1}{2(1-\lambda)}\right. \\
& \left.\frac{1}{2(1-\lambda)}+1 ; \frac{\gamma}{c_{1}} a^{4(1-\lambda)}\right), \quad \lambda<1, \quad \text { (3.15) }
\end{aligned}
$$

where $c_{2}$ is an integration constant. The restriction $\lambda<1$ above, follows from the condition for a valid integral representation of ${ }_{2} F_{1}$ described by (3.11). This means that in order to derive solutions for $\lambda>1$, we have to proceed in a different way (see Sect. 4).

In the next subsection, we shall focus on the solutions having $\lambda<1$ which suffer from a finite-distance singularity.

\subsection{Impossibility of viable solutions for $\lambda<1$}

It is straightforward to see that letting $a \rightarrow 0^{+}$in Eq. (3.15) leads to $Y \rightarrow \pm c_{2}$, which in turn implies that a collapse singularity appears within finite distance $c_{2}$. On approach to the collapse singularity, it follows from Eq. (3.1) that the density becomes divergent while Eq. (2.7) implies then that the pressure vanishes, or, diverges depending on whether $\lambda<$ 0 , or, $0<\lambda<1$, respectively.

From our experience with similar models in previous work in [5], we find that it is worth exploring the possibility of avoiding the collapse singularity by finding out if it is the only type of finite-distance singularity that solution Eq. (3.15) can exhibit, or, if instead it coexists with a big-rip type of singularity characterized by a divergent warp factor.
To examine the latter possibility, we study the behavior of the hypergeometric function as $a \rightarrow \infty$, by making use of the following transformation valid for $|\arg (-z)|<\pi$ (see [21], pp. 63-64):

$$
\begin{aligned}
& { }_{2} F_{1}(\alpha, \alpha, \alpha+1 ; z)=-\alpha(-z)^{-\alpha} \\
& \quad \sum_{n=1}^{\infty} \frac{\Gamma(\alpha+n)}{n(n !) \Gamma(\alpha)} z^{-n}+(-z)^{-\alpha}(\ln (-z)+\psi(1)-\psi(\alpha)),
\end{aligned}
$$

where $\Gamma$ is the Gamma function and $\psi$ is its logarithmic derivative [21],

$\psi(z)=\frac{d}{d z} \ln \Gamma(z)=\frac{\Gamma^{\prime}(z)}{\Gamma(z)}$.

Note that the hypergeometric function in (3.16) becomes a real valued function for those real numbers $z$ that satisfy the requirement $|\arg (-z)|<\pi$ that we mention above, thus for all non-positive real numbers $z$. The transformation of the hypergeometric function of (3.15) according to (3.16) gives,

$$
\begin{gathered}
{ }_{2} F_{1}\left(\frac{1}{2(1-\lambda)}, \frac{1}{2(1-\lambda)}, \frac{1}{2(1-\lambda)}+1 ; \frac{\gamma}{c_{1}} a^{4(1-\lambda)}\right) \\
\quad=\left(\frac{-\gamma}{c_{1}}\right)^{1 /(2(\lambda-1))} a^{-2} \times \phi(a),
\end{gathered}
$$

with,

$$
\begin{aligned}
\phi(a)= & \frac{1}{2(\lambda-1)} \sum_{n=1}^{\infty} \frac{\Gamma(1 /(2(1-\lambda))+n)}{\Gamma(1 /(2(1-\lambda))) n(n !)}\left(\frac{\gamma}{c_{1}} a^{4(1-\lambda)}\right)^{-n} \\
& +\ln \left(-\frac{\gamma}{c_{1}} a^{4(1-\lambda)}\right)+\psi(1)-\psi\left(\frac{1}{2(1-\lambda)}\right)
\end{aligned}
$$

Substituting (3.18) in (3.15) we find

$$
\begin{aligned}
\pm Y+c_{2}= & \frac{\sqrt{6}(-\gamma)^{1 /(2(\lambda-1))}}{2 \kappa_{5}} \\
& \times\left(\frac{1}{2(\lambda-1)} \sum_{n=1}^{\infty} \frac{\Gamma(1 /(2(1-\lambda))+n)}{\Gamma(1 /(2(1-\lambda))) n(n !)}\right. \\
& \times\left(\frac{\gamma}{c_{1}} a^{4(1-\lambda)}\right)^{-n}+\ln \left(-\frac{\gamma}{c_{1}} a^{4(1-\lambda)}\right) \\
& \left.+\psi(1)-\psi\left(\frac{1}{2(1-\lambda)}\right)\right) .
\end{aligned}
$$

It clearly follows from the above equation that letting $a \rightarrow$ $\infty$ and since $\lambda<1$, the series appearing in the first term inside the bracket converges to zero, but the logarithmic term that follows becomes divergent, making the RHS approach infinity. Hence we conclude that $Y \rightarrow \pm \infty$.

This shows that the singularity with a divergent warp factor shifts to infinite distance. On the other hand, the behaviors of the density and pressure of the fluid following from Eqs. (3.1) and (2.7), are $\rho \rightarrow(-\gamma)^{1 /(1-\lambda)}$ and $p \rightarrow-(-\gamma)^{1 /(1-\lambda)}$. 
The fact that for $\lambda<1$ there is only one type of finitedistance singularity, i.e a collapse singularity at $Y= \pm c_{2}$, opens-up the possibility of using a matching mechanism, similar to the one we have used for the case of a linear fluid in [4] and [5], to construct a solution free from finite-distance singularities for this range of $\lambda$. However, the problem with localizing gravity on the brane that we faced in [4], persists here as well.

This makes the choice $\lambda<1$ less promising exactly because it leads to such a compromise. We therefore avoid giving details of the matching procedure for $\lambda<1$, and focus instead on the more interesting available choice of $\lambda>1$.

We note that the specific value $\lambda=1$ will also not be studied here, as it is already analyzed in detail in our previous work [1-5]. There we showed that a linear equation of state leads to power-law behavior for the warp factor. Actually, one can also show that assuming a power-law behavior for the warp factor and density and substituting these in Eqs. (2.8),(2.9) and (2.10), forces $\lambda$ to be equal exactly equal to one.

In the next sections, we are going to show that the case of solutions with $\lambda>1$, not only offers the possibility of a regular solution but it also resolves the problem of the localization of gravity that we faced in [4] and [5].

\section{The $\lambda>1$ solutions}

In this section, we give details about the construction and regularity properties of the solutions having $\lambda>1$. This represents the most interesting case covered by our analysis.

\subsection{Construction}

For values of $\lambda$ greater than one, the requirement for the integral representation previously met in the case $\lambda<1$, is no longer satisfied and we therefore have to elaborate further on the integral in the LHS of Eq. (3.5) to get a valid representation of a hypergeometric function. This involves applying first integration by parts from which we find,

$$
\begin{aligned}
\int & \frac{a}{\left(c_{1}-\gamma a^{4(1-\lambda)}\right)^{1 /(2(1-\lambda))}} d a \\
= & \frac{a^{2}}{2}\left(c_{1}-\gamma a^{4(1-\lambda)}\right)^{1 /(2(\lambda-1))}- \\
& -\gamma \int a^{5-4 \lambda}\left(c_{1}-\gamma a^{4(1-\lambda)}\right)^{(3-2 \lambda) /(2(\lambda-1))} d a
\end{aligned}
$$

and then expressing the new integral on the RHS of the above equation as an integral representation of a hypergeometric function, with a procedure similar to the one we did in Sect. 3.1, for $\lambda<1$. While performing such a procedure and in order to satisfy restriction (3.11), we have to confine the range of $\lambda$ to values greater than $3 / 2$. We arrive at the following solution

$$
\begin{aligned}
\pm Y+c_{2}= & \frac{\sqrt{6}}{\kappa_{5}}\left(\frac{a^{2}}{2}\left(c_{1}-\gamma a^{4(1-\lambda)}\right)^{1 /(2(\lambda-1))}\right. \\
& -\frac{\gamma c_{1}^{(3-2 \lambda) /(2(\lambda-1))}}{2(3-2 \lambda)} a^{2(3-2 \lambda)} \\
& \times{ }_{2} F_{1}\left(\frac{3-2 \lambda}{2(1-\lambda)}, \frac{3-2 \lambda}{2(1-\lambda)}, \frac{3-2 \lambda}{2(1-\lambda)}+1 ;\right. \\
& \left.\left.\frac{\gamma}{c_{1}} a^{4(1-\lambda)}\right)\right),
\end{aligned}
$$

In (4.2), we have used the following integral representation

$$
\begin{aligned}
{ }_{2} F_{1} & \left(\frac{3-2 \lambda}{2(1-\lambda)}, \frac{3-2 \lambda}{2(1-\lambda)}, \frac{3-2 \lambda}{2(1-\lambda)}+1 ; \frac{\gamma}{c_{1}} a^{4(1-\lambda)}\right)= \\
= & \frac{2(1-\lambda)}{3-2 \lambda} \int_{0}^{1} w^{(3-2 \lambda) /(2(1-\lambda))-1} \\
& \times\left(1-\frac{\gamma}{c_{1}} a^{4(1-\lambda)} w\right)^{-(3-2 \lambda) /(2(1-\lambda))} d w .
\end{aligned}
$$

We note that for $1<\lambda<3 / 2$, the parameters of the hypergeometric function become negative and restriction (3.11) is not satisfied. This means that if we wish to find solutions for such values of $\lambda$, we cannot accept the integral representation of the hypergeometric function as implied by Eq. (4.2).

Finding a solution for $1<\lambda<3 / 2$, becomes somewhat more intricate. The reason is, that in order to obtain a valid representation of a hypergeometric function, we have to keep track with condition (3.11) that requires the $\lambda$-dependent parameters of the hypergeometric function, to be positive. This results in confining $\lambda$ to appropriate intervals, which in turn amounts to slicing the interval $(1,3 / 2)$ into subintervals.

To proceed, we consider separately ranges of values of $\lambda$ such as $5 / 4<\lambda<3 / 2,7 / 6<\lambda<5 / 4$ and in general $(n+1) / n<\lambda<(n-1) /(n-2)$, with $n=2 k$ and $k$ a positive integer. As we consider values of $\lambda$ closer and closer to one, we construct our solution by taking the integral that was expressed as a representation of a hypergeometric function in one interval, say $((n-1) /(n-2),(n-3) /(n-4))$, and performing one more step of integration by parts to derive a solution for its left-adjacent interval, that is $((n+1) / n,(n-$ 1) $/(n-2))$.

We end up with solutions expressed by hypergeometric functions of the form ${ }_{2} F_{1}(\alpha, \alpha, \alpha+1 ; z)$, where $\alpha=(n-$ $n \lambda+1) /(2(1-\lambda))$, and $z=\left(\gamma / c_{1}\right) a^{4(1-\lambda)}$, and valid for $(n+1) / n<\lambda<(n-1) /(n-2)$.

We therefore see that as we move from one interval $((n-1) /(n-2),(n-3) /(n-4))$ to the next $((n-$ $1) /(n-2),(n-3) /(n-4))$, we obtain a solution expressed by a hypergeometric function that has a different parameter $\alpha$ every time, but which maintains the same argument $z=\left(\gamma / c_{1}\right) a^{4(1-\lambda)}$, with $\lambda>1$. 
Each of these hypergeometric series is convergent for $|z|<1$, which in our case translates to $a^{4(\lambda-1)}>-\gamma / c_{1}$, where the bound $-\gamma / c_{1}$ can of course be made arbitrarily large.

In the solutions we obtained through this method, we shall consider asymptotic behaviors of the form $a \rightarrow \infty$, ensuring in this way convergence of all hypergeometric series that appear, independently of the interval of validity of the solutions.

To see this clearly, consider for example the range $5 / 4<$ $\lambda<3 / 2$. We take the integral on the RHS of Eq. (4.1) and perform one more time integration by parts and find

$$
\begin{aligned}
\int & a^{5-4 \lambda}\left(c_{1}-\gamma a^{4(1-\lambda)}\right)^{(3-2 \lambda) /(2(\lambda-1))} d a \\
= & \frac{a^{2(3-2 \lambda)}}{2(3-2 \lambda)}\left(c_{1}-\gamma a^{4(1-\lambda)}\right)^{(3-2 \lambda) /(2(\lambda-1))}- \\
& -\gamma \int a^{9-8 \lambda}\left(c_{1}-\gamma a^{4(1-\lambda)}\right)^{(5-4 \lambda) /(2(\lambda-1))} d a .
\end{aligned}
$$

We then express the integral on the RHS of the above equation in terms of a hypergeometric function which leads us to the following solution valid for $5 / 4<\lambda<3 / 2$,

$$
\begin{aligned}
& \pm Y+c_{2}=\frac{\sqrt{6}}{\kappa_{5}}\left(\frac{a^{2}}{2}\left(c_{1}-\gamma a^{4(1-\lambda)}\right)^{1 /(2(\lambda-1))}\right. \\
& -\frac{\gamma}{2(3-2 \lambda)} a^{2(3-2 \lambda)}\left(c_{1}-\gamma a^{4(1-\lambda)}\right)^{(3-2 \lambda) /(2(\lambda-1))} \\
& \quad+\frac{\gamma^{2} c_{1}^{(5-4 \lambda) /(2(\lambda-1))}}{2(5-4 \lambda)} a^{2(5-4 \lambda)} \\
& \left.\quad \times 2 F_{1}\left(\frac{5-4 \lambda}{2(1-\lambda)}, \frac{5-4 \lambda}{2(1-\lambda)}, \frac{5-4 \lambda}{2(1-\lambda)}+1 ; \frac{\gamma}{c_{1}} a^{4(1-\lambda)}\right)\right) .
\end{aligned}
$$

We note again that for $\lambda<5 / 4$ the parameters of the hypergeometric function are negative and this means that we have to go one more step further and perform integration by parts on the integral on the RHS of Eq. (4.4). Following the same line of thinking, we see that to get a solution we have to refine the values of $\lambda$ in the interval $7 / 6<\lambda<5 / 4$. The solution we find reads

$$
\begin{aligned}
\pm Y+c_{2}= & \frac{\sqrt{6}}{\kappa_{5}}\left(\frac{a^{2}}{2}\left(c_{1}-\gamma a^{4(1-\lambda)}\right)^{1 /(2(\lambda-1))}\right. \\
& -\frac{\gamma}{2(3-2 \lambda)} a^{2(3-2 \lambda)}\left(c_{1}-\gamma a^{4(1-\lambda)}\right)^{(3-2 \lambda) /(2(\lambda-1))} \\
& +\frac{\gamma^{2}}{2(5-4 \lambda)} a^{2(5-4 \lambda)}\left(c_{1}-\gamma a^{4(1-\lambda)}\right)^{(5-4 \lambda) /(2(\lambda-1))}- \\
& -\frac{\gamma^{3} c_{1}^{(7-6 \lambda) /(2(\lambda-1))}}{2(7-6 \lambda)} a^{2(7-6 \lambda)} \\
& \left.\times{ }_{2} F_{1}\left(\frac{7-6 \lambda}{2(1-\lambda)}, \frac{7-6 \lambda}{2(1-\lambda)}, \frac{7-6 \lambda}{2(1-\lambda)}+1 ; \frac{\gamma}{c_{1}} a^{4(1-\lambda)}\right)\right) .
\end{aligned}
$$

In a similar way, we can generalize the previous results for $(n+1) / n<\lambda<(n-1) /(n-2)$, with $n=2 k$ and $k$ a positive integer, and perform integration by parts $n / 2$ times. The solution then takes the form,

$$
\begin{aligned}
\pm Y+c_{2}= & \frac{\sqrt{6}}{\kappa_{5}}\left(\frac{a^{2}}{2}\left(c_{1}-\gamma a^{4(1-\lambda)}\right)^{1 /(2(\lambda-1))}\right. \\
& -\frac{\gamma}{2(3-2 \lambda)} a^{2(3-2 \lambda)}\left(c_{1}-\gamma a^{4(1-\lambda)}\right)^{(3-2 \lambda) /(2(\lambda-1))} \\
& +\frac{\gamma^{2}}{2(5-4 \lambda)} a^{2(5-4 \lambda)}\left(c_{1}-\gamma a^{4(1-\lambda)}\right)^{(5-4 \lambda) /(2(\lambda-1))}+\ldots \\
& +\frac{(-\gamma)^{n / 2-1}}{2((n-1)-(n-2) \lambda)} a^{2((n-1)-(n-2) \lambda)} \\
& \times\left(c_{1}-\gamma a^{4(1-\lambda)}\right)^{((n-1)-(n-2) \lambda) /(2(\lambda-1))} \\
& +\frac{(-\gamma)^{n / 2} c_{1}^{((n+1)-n \lambda) /(2(\lambda-1))}}{2((n+1)-n \lambda)} a^{2((n+1)-n \lambda)} \\
& \times{ }_{2} F_{1}\left(\frac{(n+1)-n \lambda}{2(1-\lambda)}, \frac{(n+1)-n \lambda}{2(1-\lambda)},\right. \\
& \left.\left.\times \frac{(n+1)-n \lambda}{2(1-\lambda)}+1 ; \frac{\gamma}{c_{1}} a^{4(1-\lambda)}\right)\right) .
\end{aligned}
$$

Direct substitution of the above equation in the field equations (2.8)-(2.9) verifies that this is indeed the general form of solution for $(n+1) / n<\lambda<(n-1) /(n-2)$. For convenience, we can write the above equation as follows

$$
\begin{aligned}
& \pm Y+c_{2}=\frac{\sqrt{6}}{\kappa_{5}} \sum_{s=0}^{n / 2-1} \frac{(-\gamma)^{s}}{2(1-2 s(\lambda-1))} \\
& \quad \times\left(c_{1} a^{4(\lambda-1)}-\gamma\right)^{1 /(2(\lambda-1))-s} \\
& +\frac{(-\gamma)^{n / 2}\left(c_{1}\right)^{((n+1)-n \lambda) /(2(\lambda-1))}}{2((n+1)-n \lambda)} a^{2((n+1)-n \lambda)} \\
& \quad \times{ }_{2} F_{1}\left(\frac{(n+1)-n \lambda}{2(1-\lambda)}, \frac{(n+1)-n \lambda}{2(1-\lambda)}, \frac{(n+1)-n \lambda}{2(1-\lambda)}\right. \\
& \left.\left.\quad+1 ; \frac{\gamma}{c_{1}} a^{4(1-\lambda)}\right)\right)
\end{aligned}
$$

We next show that $\lambda>1$ is exactly the appropriate range that provides us with regular solutions free from finite-distance singularities. Based on this type of solution, one can construct a regular braneworld model with matter that satisfies the strong and null energy conditions and which also successfully localizes gravity on the flat brane.

\subsection{Regularity}

In this subsection we study the behaviors of solutions for $\lambda>1$ and prove that they are free of finite $Y$-distance singularities. As we saw previously, the analysis for this range of $\lambda$ leads to three cases, each one defined by a refinement in the range of $\lambda$ and an implicit form of solution for the warp factor $a(Y)$ :

I. For $\lambda=(n+1) / n, n=2 k$ and $k$ a positive integer, $a(Y)$ is given by Eq. (3.7).

II. For $\lambda>3 / 2, a(Y)$ is given by Eq. (4.2). 
III. For $1<\lambda<3 / 2$ and excepting values of $\lambda$ that fall in case I, the solution for $a(Y)$ is given by Eq. (4.8) and is valid inside open subintervals of the form $((n+1) / n,(n-$ $1) /(n-2))$ with $n=2 k$ and $k$ a positive integer such that $k \geq 2$.

We begin our study with the first and simplest case I. If we let $a \rightarrow 0^{+}$in (3.7), then the RHS will approach infinity as a result of the logarithmic term that appears there, which in turn implies that $Y \rightarrow \pm \infty$. This means that the collapse singularity that we encountered previously for $\lambda<1$, shifts, in this new case, to infinite distance.

For the asymptotic behaviors of $\rho$ and $p$ for $Y \rightarrow \pm \infty$, we can deduce from Eq. (3.1) and Eq. (2.7), that they are $\rho \rightarrow(-\gamma)^{1 /(1-\lambda)}$ and $p \rightarrow-(-\gamma)^{1 /(1-\lambda)}$.

On the other hand, if we let $a \rightarrow \infty$, we see that all terms on the RHS of (3.7) diverge, leading again to $Y \rightarrow \pm \infty$, while as it follows from Eqs. (3.1) and (2.7), the density and pressure vanish asymptotically. The solution described by case $I$ ) is therefore free from finite-distance singularities.

We continue with the study of case II. We note that since in Eq. (4.2) the power of the argument of the hypergeometric function is, in this case, negative, the most straightforward asymptotic behavior implied by Eq. (4.2), is that of $a \rightarrow \infty$ that makes the argument approach zero thus leading to a convergent hypergeometric series. Allowing $a \rightarrow \infty$, results in the divergence of the first term on the RHS of Eq. (4.2) and leads to $Y \rightarrow \pm \infty$. The density and pressure, on the other hand, vanish asymptotically.

Having escaped the emergence of a finite-distance singularity with a divergent warp factor, we proceed by researching the possibility of a collapse finite-distance singularity characterized by $a \rightarrow 0^{+}$. As was done earlier, we have to first transform the hypergeometric function of Eq. (4.2) using (3.16) and then substitute back in Eq. (4.2) to find the implicit form of solution for the warp factor. We end up with the following result,

$$
\begin{aligned}
\pm & +c_{2}=\frac{\sqrt{6}}{\kappa_{5}}\left(\frac{a^{2}}{2}\left(c_{1}-\gamma a^{4(1-\lambda)}\right)^{1 /(2(\lambda-1))}\right. \\
& +\frac{(-\gamma)^{1 /(2(\lambda-1))}}{4(\lambda-1)} \sum_{n=1}^{\infty} \frac{\Gamma((3-2 \lambda) /(2(1-\lambda))+n)}{\Gamma((3-2 \lambda) /(2(1-\lambda))) n(n !)} \\
& \times\left(\frac{\gamma}{c_{1}} a^{4(1-\lambda)}\right)^{-n}+\frac{(-\gamma)^{1 /(2(\lambda-1))}}{2(3-2 \lambda)}\left(\ln \left(-\frac{\gamma}{c_{1}} a^{4(1-\lambda)}\right)\right. \\
& \left.\left.+\psi(1)-\psi\left(\frac{3-2 \lambda}{2(1-\lambda)}\right)\right)\right) .
\end{aligned}
$$

Putting $a \rightarrow 0^{+}$in the above equation, gives $Y \rightarrow \pm \infty$ which means that the collapse type of singularity is located at infinite distance. Also, from Eqs. (3.1) and (2.7), it follows that this behavior occurs with $\rho \rightarrow(-\gamma)^{1 /(1-\lambda)}$ and $p \rightarrow$ $-(-\gamma)^{1 /(1-\lambda)}$.
Finally, the remaining case III is very similar to case II with the transformation of Eq. (4.8) according to Eq. (3.16) being in this case,

$$
\begin{aligned}
& \pm Y+c_{2}=\frac{\sqrt{6}}{2 \kappa_{5}}\left(\sum_{s=0}^{n / 2-1} \frac{(-\gamma)^{s}}{1-2 s(\lambda-1)}\left(c_{1} a^{4(\lambda-1)}-\gamma\right)^{1 /(2(\lambda-1))-s}\right. \\
& +(-\gamma)^{1 /(2(\lambda-1))}\left(\frac{1}{2(\lambda-1)} \sum_{n=1}^{\infty} \frac{\Gamma(((n+1)-n \lambda) /(2(1-\lambda))+n)}{\Gamma(((n+1)-n \lambda) /(2(1-\lambda))) n(n !)}\right. \\
& \quad \times\left(\frac{\gamma}{c_{1}} a^{4(1-\lambda)}\right)^{-n}+\frac{1}{(n+1)-n \lambda}\left(\ln \left(-\frac{\gamma}{c_{1}} a^{4(1-\lambda)}\right)\right. \\
& \left.\left.\left.\quad+\psi(1)-\psi\left(\frac{(n+1)-n \lambda}{2(1-\lambda)}\right)\right)\right)\right) .
\end{aligned}
$$

The procedure of deriving the asymptotic behaviors implied by Eq. (4.8) follows along the same steps we performed for case II, and we encounter again the same asymptotic behaviors, namely,

$$
\begin{aligned}
& a \rightarrow \infty \text { and } \rho \rightarrow 0, p \rightarrow 0, \quad \text { as } Y \rightarrow \pm \infty \\
& a \rightarrow 0^{+} \text {and } \rho \rightarrow(-\gamma)^{1 /(1-\lambda)}, p \rightarrow-(-\gamma)^{1 /(1-\lambda)}, \text { as } \\
& Y \rightarrow \pm \infty
\end{aligned}
$$

We have therefore shown that all three cases lead to $\lambda$ solutions, with $\lambda>1$ that are regular in the sense of being free from finite-distance singularities. In Sects. 6, 7, we shall further explore other characteristics of these solutions, such as the issue of localization of gravity on the brane and the matching conditions, that make these solutions viable in the sense discussed in the Introduction.

However, before dealing with these more complicated issues in the general case, we shall present in the next section a worked example for the simplest possible among all the solutions in the 1-parameter family of $\lambda$-solutions, namely the solution for $\lambda=3 / 2$. We shall show that this solution shares the same basic features with all other solutions in the family, and hence may offer clear insight into the generic behaviour of the whole set.

\section{Matching and localization: a special case}

In this section, we focus on the solution for $\lambda=3 / 2$ given in implicit form by Eq. (3.6). Looking at Eq. (3.6), it is straightforward to see that it leads to the asymptotic behaviors described by Eqs. (4.11)-(4.12), once we input $\lambda=3 / 2$ when $\lambda$ appears there. Our plan is to combine the two branches of solutions of Eq. (3.6) and construct a matching solution that leads to a finite Planck mass, thus rectifying the problem of localization of gravity on the brane we had with previous models in [4] and [5]. 


\subsection{Derivatives}

We start by writing individually the two branches of solutions of Eq. (3.6), these are:

$$
\begin{aligned}
& Y^{+}=h_{+}(a)=\frac{\sqrt{6}}{\kappa_{5}}\left(\frac{c_{1}^{+}}{2} a^{2}-\gamma \ln a\right)+C_{2}^{+} \\
& Y^{-}=h_{-}(a)=\frac{\sqrt{6}}{\kappa_{5}}\left(-\frac{c_{1}^{-}}{2} a^{2}+\gamma \ln a\right)+C_{2}^{-},
\end{aligned}
$$

where $Y^{ \pm}$denote the solutions we find for $Y$ using the $( \pm)$ sign in Eq. (3.6) and are expressed for convenience as functions, $h_{ \pm}(a)$, of the warp factor, while $c_{1}^{ \pm}, C_{2}^{ \pm}$are the values of $c_{1}$ and $\mp c_{2}$, respectively, on the ( \pm ) branch of $Y$. Taking the first and second derivative of $h_{+}(a)$ we find

$$
\begin{aligned}
& h_{+}^{\prime}(a)=\frac{\sqrt{6}}{\kappa_{5}}\left(\frac{c_{1}^{+} a^{2}-\gamma}{a}\right) \\
& h_{+}^{\prime \prime}(a)=\frac{\sqrt{6}}{\kappa_{5}} \frac{c_{1}^{+}}{a^{2}}\left(a^{2}+\frac{\gamma}{c_{1}^{+}}\right) .
\end{aligned}
$$

Now, since $\gamma<0$ and $c_{1}^{+}>0$, the first derivative is strictly positive, while the second derivative changes from negative on $\left(0, \sqrt{-\gamma / c_{1}^{+}}\right)$to positive on $\left(\sqrt{-\gamma / c_{1}^{+}}, \infty\right)$ and crosses zero at $a=\sqrt{-\gamma / c_{1}^{+}}$, making the point $\left(\sqrt{-\gamma / c_{1}^{+}},-\sqrt{6} / \kappa_{5}\left(\gamma / 2+\gamma \ln \sqrt{-\gamma / c_{1}^{+}}\right)+C_{2}^{+}\right)$an inflection point of the graph of $h_{+}$.

Calculating the first two derivatives of $h_{-}(a)$, on the other hand, gives similar results the only difference being that the signs of the derivatives are the opposites of the ones of $h_{+}$ given in Eqs. (5.3)-(5.4) above. This means that $h_{-}^{\prime}$ is strictly negative and $h_{-}^{\prime \prime}$ changes from positive on $\left(0, \sqrt{-\gamma / c_{1}^{-}}\right)$to negative on $\left(\sqrt{-\gamma / c_{1}^{-}}, \infty\right)$ with $\left(\sqrt{-\gamma / c_{1}^{-}}, \sqrt{6} / \kappa_{5}(\gamma / 2+\right.$ $\left.\gamma \ln \sqrt{-\gamma / c_{1}^{-}}\right)+C_{2}^{-}$) being an inflection point of the graph of $h_{-}$.

\subsection{Matching at the inflection point}

As we saw in Sect. 3, convergence of the power series expansion of the integral of the hypergeometric series given by Eq. (3.15) is effected as long as $z$ is not a real number bigger than unity.

This means that for $\lambda=3 / 2$, we must have,

$a>\left|\frac{\gamma}{c_{1}}\right|^{1 / 2}$,

but as we have already remarked, convergence may be extended by analytic continuation to regions far beyond that, where the opposite inequality will be true. The important thing is that because of this, we can match the two branches at the inflection point of their graphs found above, because the convergence of the matching solution is only consistent in that case.

A natural assumption is that the warp factor should be continuous there so

$\sqrt{\frac{-\gamma}{c_{1}^{+}}}=\sqrt{\frac{-\gamma}{c_{1}^{-}}}, \quad$ or, $\quad c_{1}^{+}=c_{1}^{-}=c_{1}$.

Also, the inflection point should have to the same $Y$ coordinate through the two branches which means that

$h_{+}\left(\sqrt{\frac{-\gamma}{c_{1}}}\right)=h_{-}\left(\sqrt{\frac{-\gamma}{c_{1}}}\right)$

and yields the following relation between $C_{2}^{+}$and $C_{2}^{-}$,

$$
\begin{aligned}
& -\frac{\sqrt{6}}{\kappa_{5}}\left(\frac{\gamma}{2}+\gamma \ln \sqrt{\frac{-\gamma}{c_{1}}}\right)+C_{2}^{+} \\
& =\frac{\sqrt{6}}{\kappa_{5}}\left(\frac{\gamma}{2}+\gamma \ln \sqrt{\frac{-\gamma}{c_{1}}}\right)+C_{2}^{-},
\end{aligned}
$$

or,

$C_{2}^{+}=\frac{\sqrt{6}}{\kappa_{5}}\left(\gamma+2 \gamma \ln \sqrt{\frac{-\gamma}{c_{1}}}\right)+C_{2}^{-}$.

The matching graphs that we obtain in this way, have an axis of symmetry at $Y=Y_{s}$, with $Y_{s}$ given by

$$
\begin{aligned}
Y_{s} & =h_{ \pm}\left(\sqrt{-\gamma / c_{1}}\right)=-\frac{\sqrt{6}}{\kappa_{5}}\left(\frac{\gamma}{2}+\gamma \ln \sqrt{\frac{-\gamma}{c_{1}}}\right)+C_{2}^{+} \\
& =\frac{\sqrt{6}}{\kappa_{5}}\left(\frac{\gamma}{2}+\gamma \ln \sqrt{\frac{-\gamma}{c_{1}}}\right)+C_{2}^{-}
\end{aligned}
$$

and designating the position of the brane. For simplicity we can place the brane at $Y=0$ by putting $Y_{S}=0$ in (5.10) and calculating the values of $C_{2}^{ \pm}$that make this possible. We find

$C_{2}^{+}=\frac{\sqrt{6}}{\kappa_{5}}\left(\frac{\gamma}{2}+\gamma \ln \sqrt{\frac{-\gamma}{c_{1}}}\right)=-C_{2}^{-}$.

The matching solution described by Eq. (5.1) and (5.2) that satisfies the above boundary condition can be written as

$$
\begin{aligned}
|Y| & =\frac{\sqrt{6}}{\kappa_{5}}\left(-\frac{c_{1}}{2} a^{2}+\gamma \ln a-\frac{\gamma}{2}-\gamma \ln \sqrt{\frac{-\gamma}{c_{1}}}\right), \\
0 & <a \leq \sqrt{\frac{-\gamma}{c_{1}}} .
\end{aligned}
$$

We note that $\rho$ is well defined and continuous at the location of the brane $Y=0$ and its value is

$\rho\left(\sqrt{\frac{-\gamma}{c_{1}}}\right) \equiv \rho(0)=\frac{1}{4 \gamma^{2}}$,

where $\rho(0)$ denotes $\rho(Y=0)$ and it is an abbreviation of $\rho\left(h_{ \pm}^{-1}\left(\sqrt{-\gamma / c_{1}}\right)\right)$.

In Figs. 1, 2 and 3, we depict the graphs of $h_{+}, h_{-}$and the matching branches, respectively. In Fig. 4, we have rotated 


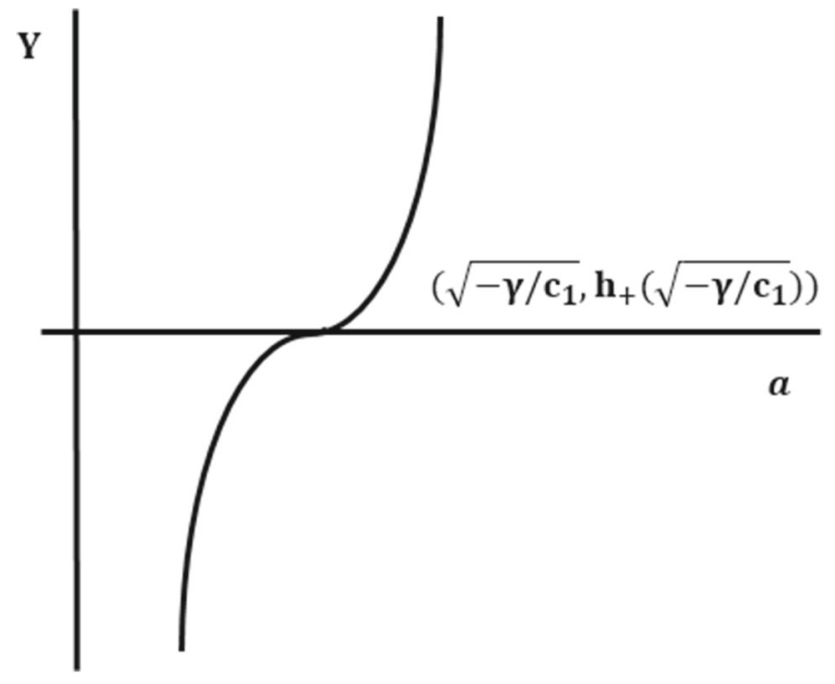

Fig. 1 Graph of $h_{+}(a)$

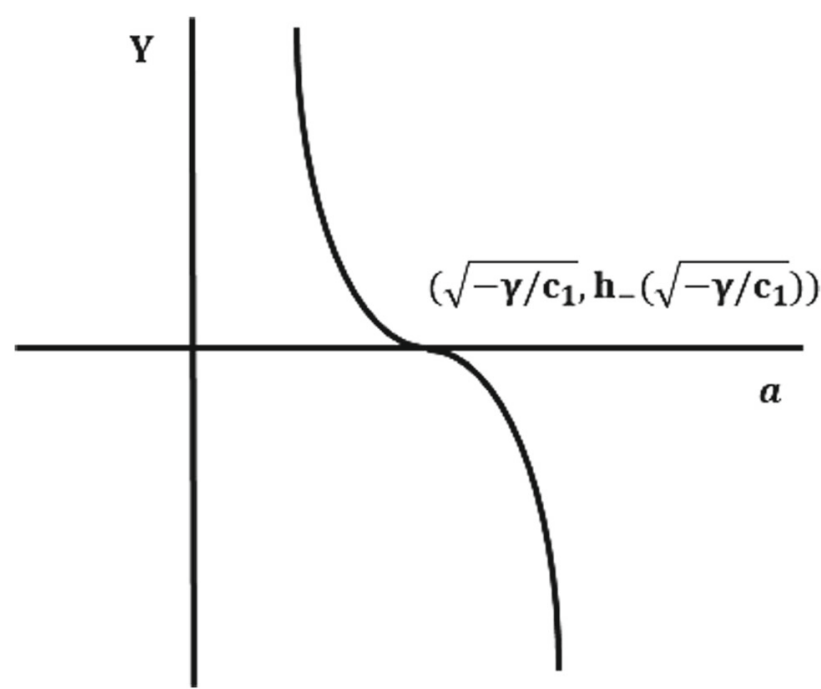

Fig. 2 Graph of $h_{-}(a)$

the axes of Fig. 3, to gain a more convenient view of the evolution of the warp factor as a function of $Y$. The reason for constructing the matching solution by keeping the solid lines instead of the dashed ones in Fig. 3, is to obtain a finite Planck mass, as we show in the analysis below.

Next, we take into account the jump of the derivative of the warp factor across the brane and find, for our type of geometry, the following junction condition

$a^{\prime}\left(0^{+}\right)-a^{\prime}\left(0^{-}\right)=2 a^{\prime}\left(0^{+}\right)=-\kappa_{5}^{2} \frac{f(\rho(0)) a(0)}{3}$,

where $f(\rho(0))$ is the tension of the brane. Using Eq. (2.8), in the above equation we can derive the relation between the brane tension and the density, this reads

$f(\rho(0))=-\frac{\sqrt{6 \rho(0)}}{\kappa_{5}}=\frac{\sqrt{6}}{2 \kappa_{5} \gamma}$.

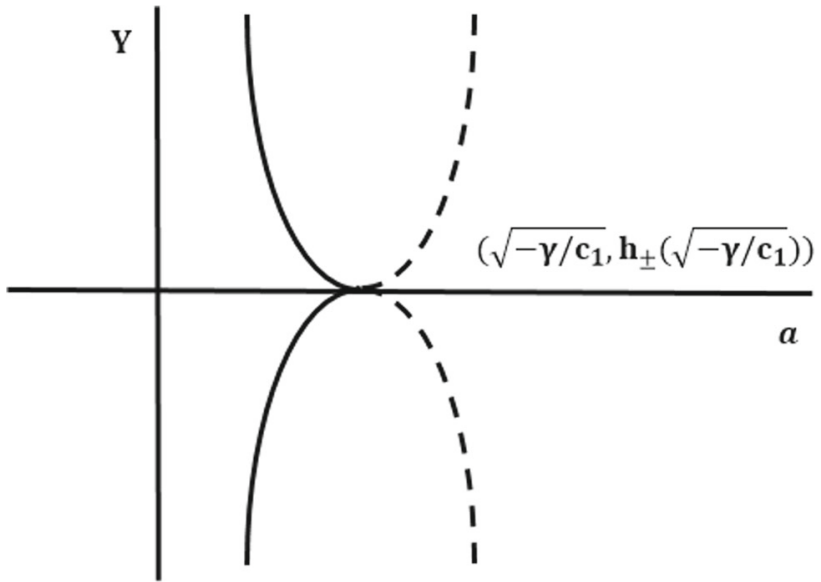

Fig. 3 Matching graphs at the inflection point

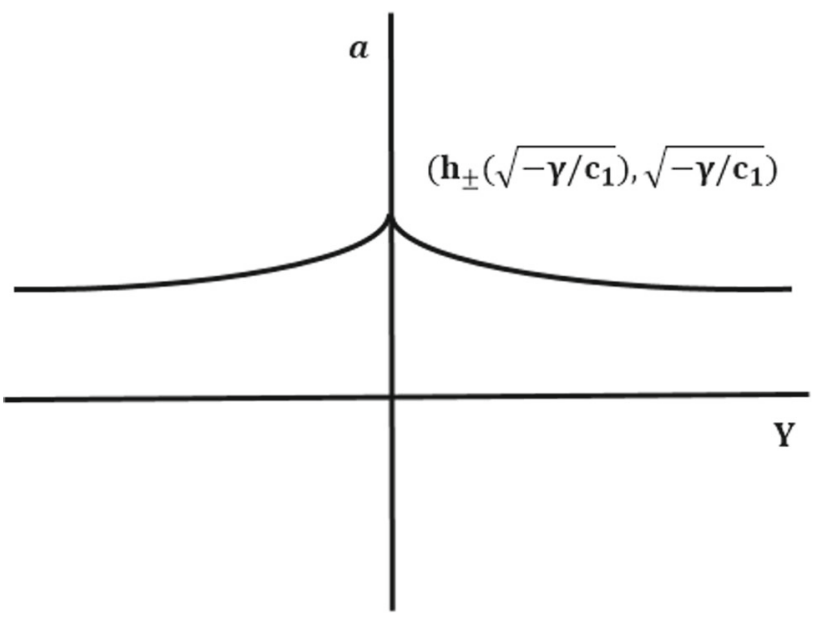

Fig. 4 Graph of the warp factor as a function of $Y$, for $\lambda=3 / 2$

\subsection{Localization}

Finally, we calculate the Planck mass for the matching solution. The value of the four-dimensional Planck mass, $M_{p}^{2}=8 \pi / \kappa$, is determined by the following integral (see $[4,5,23])$,

$\frac{\kappa_{5}^{2}}{\kappa}=\int_{-Y_{c}}^{Y_{c}} a^{2}(Y) d Y$

For the solution of Eq. (5.12), the behaviour of $a^{2}$ as $Y \rightarrow$ $-\infty$ is

$a^{2} \sim e^{-\left(\sqrt{6} \kappa_{5} /(3 \gamma)\right) Y}$,

and using the symmetry of Eq. (5.12), we can write the above integral in the following form

$$
\begin{aligned}
\int_{-Y_{c}}^{Y_{c}} a^{2} d Y & =2 \int_{-Y_{c}}^{0} a^{2} d Y \sim 2 \int_{-Y_{c}}^{0} e^{-\left(\sqrt{6} \kappa_{5} /(3 \gamma)\right) Y} d Y \\
& =-\sqrt{6} \frac{\gamma}{\kappa_{5}}\left(1-e^{\left(\sqrt{6} \kappa_{5} /(3 \gamma)\right) Y_{c}}\right) .
\end{aligned}
$$


In the limit $Y_{c} \rightarrow \infty$ and since $\gamma<0$, we see that the Planck mass remains finite and is proportional to

$-\sqrt{6} \frac{\gamma}{\kappa_{5}}$.

Summarizing the case of study of $\lambda=3 / 2$, we have constructed a regular matching solution that satisfies both the null and strong energy conditions and at the same time successfully localizes gravity on the brane.

\section{Matching: the general case}

Now, we generalize the matching mechanism we constructed in the previous section for the case of $\lambda=3 / 2$, to cover the broader case of $\lambda>1$. As mentioned before, the two cases share many common features and we can use the example of $\lambda=3 / 2$ as a guide to the most complicated case of $\lambda>1$.

We begin by writing separately the two solutions we get for $\lambda=(n+1) / n, n=2 k, \mathrm{k}$ a positive integer given by Eq. (3.7), we have

$$
\begin{aligned}
Y^{+}= & h_{+}(a)=\frac{\sqrt{6}}{\kappa_{5}}\left(\sum_{s=0}^{k-1} \frac{k !}{(k-s) ! s !} \frac{\left(c_{1}^{+}\right)^{k-s}}{2-2 s / k}\right. \\
& \left.\times a^{2-2 s / k}(-\gamma)^{s}+(-\gamma)^{k} \ln a\right)+C_{2}^{+} \\
Y^{-}= & h_{-}(a)=-\frac{\sqrt{6}}{\kappa_{5}}\left(\sum_{s=0}^{k-1} \frac{k !}{(k-s) ! s !} \frac{\left(c_{1}^{-}\right)^{k-s}}{2-2 s / k}\right. \\
& \left.\times a^{2-2 s / k}(-\gamma)^{s}+(-\gamma)^{k} \ln a\right)+C_{2}^{-},
\end{aligned}
$$

where the notation of $Y^{ \pm}, c_{1}^{ \pm}$and $C_{2}^{ \pm}$represents, as in Sect. 5, the values of the variables/constants of the $( \pm)$ choice of solution respectively.

To evaluate easily the first two derivatives of $h_{+}$, we note from Eq. (3.5) that $h_{+}^{\prime}$ is equal to the integrand function that appears there. After some manipulation we can rewrite the integrand function as $\left(c_{1}^{+} a^{1 / k}-\gamma a^{-1 / k}\right)^{k}$ and then differentiate once more to evaluate $h_{+}^{\prime \prime}$, we obtain

$h_{+}^{\prime}(a)=\frac{\sqrt{6}}{\kappa_{5}}\left(c_{1}^{+} a^{1 / k}-\gamma a^{-1 / k}\right)^{k}$
$h_{+}^{\prime \prime}(a)=\frac{\sqrt{6}}{\kappa_{5}} a^{-1 / k-1}\left(c_{1}^{+} a^{1 / k}-\gamma a^{-1 / k}\right)^{k-1}\left(c_{1}^{+} a^{2 / k}+\gamma\right)$.

Since $\gamma<0$ and $c_{1}^{+}>0, h_{+}^{\prime}$ is always positive and $h_{+}^{\prime \prime}$ changes from negative on $\left(0,\left(-\gamma / c_{1}^{+}\right)^{k / 2}\right)$ to positive on $\left(\left(-\gamma / c_{1}^{+}\right)^{k / 2}, \infty\right)$, so that the point

$\left(\left(-\gamma / c_{1}^{+}\right)^{k / 2}, h_{+}\left(\left(-\gamma / c_{1}^{+}\right)^{k / 2}\right)\right)$ is an inflection point of the graph of $h_{+}$. As in Sect. 5, we can deduce the behavior of the first two derivatives of $h_{-}$from those of $h_{+}$since they are opposites.

As in the previous section, we are allowed to join the two solutions of Eqs. (6.1)-(6.2) at their common inflection point. This implies that we take,

$c_{1}^{+}=c_{1}^{-}=c_{1}$,

and

$h_{+}\left(\left(\frac{-\gamma}{c_{1}}\right)^{k / 2}\right)=h_{-}\left(\left(\frac{-\gamma}{c_{1}}\right)^{k / 2}\right)$.

The latter equation reveals the relation between the two constants $C_{2}^{ \pm}$

$$
\begin{aligned}
C_{2}^{+}= & -\frac{2 \sqrt{6}}{\kappa_{5}}\left(\sum_{s=0}^{k-1} \frac{k !}{(k-s) ! s !} \frac{(-\gamma)^{k}}{2-2 s / k}\right. \\
& \left.+(-\gamma)^{k} \ln \left(\frac{-\gamma}{c_{1}}\right)^{k / 2}\right)+C_{2}^{-} .
\end{aligned}
$$

The matching graphs have an axis of symmetry at $Y=Y_{S}$, with

$$
\begin{aligned}
Y_{s}= & \left.h_{ \pm}\left(\left(-\gamma / c_{1}\right)^{k / 2}\right)\right) \\
= & \frac{\sqrt{6}}{\kappa_{5}}\left(\sum_{s=0}^{k-1} \frac{k !}{(k-s) ! s !} \frac{(-\gamma)^{k}}{2-2 s / k}\right. \\
& \left.+(-\gamma)^{k} \ln \left(\frac{-\gamma}{c_{1}}\right)^{k / 2}\right)+C_{2}^{+} \\
= & -\frac{\sqrt{6}}{\kappa_{5}}\left(\sum_{s=0}^{k-1} \frac{k !}{(k-s) ! s !} \frac{(-\gamma)^{k}}{2-2 s / k}\right. \\
& \left.+(-\gamma)^{k} \ln \left(\frac{-\gamma}{c_{1}}\right)^{k / 2}\right)+C_{2}^{-},
\end{aligned}
$$

hence $Y_{S}$ is where we can position the brane. To simplify our expressions we take again $Y_{S}=0$, which means that the arbitrary constants $C_{2}^{ \pm}$are set to the values

$$
\begin{aligned}
C_{2}^{+}= & -\frac{\sqrt{6}}{\kappa_{5}}\left(\sum_{s=0}^{k-1} \frac{k !}{(k-s) ! s !} \frac{(-\gamma)^{k}}{2-2 s / k}\right. \\
& \left.+(-\gamma)^{k} \ln \left(\frac{-\gamma}{c_{1}}\right)^{k / 2}\right)=-C_{2}^{-} .
\end{aligned}
$$

The matching solution described by Eqs. (6.1) and (6.2) that satisfies the boundary condition (6.9) then takes the form

$$
\begin{aligned}
|Y|= & \frac{\sqrt{6}}{\kappa_{5}}\left(\sum_{s=0}^{k-1} \frac{k !}{(k-s) ! s !(2-2 s / k)}\right. \\
& \times\left(-c_{1}^{k-s} a^{2-2 s / k}(-\gamma)^{s}+(-\gamma)^{k}\right)
\end{aligned}
$$




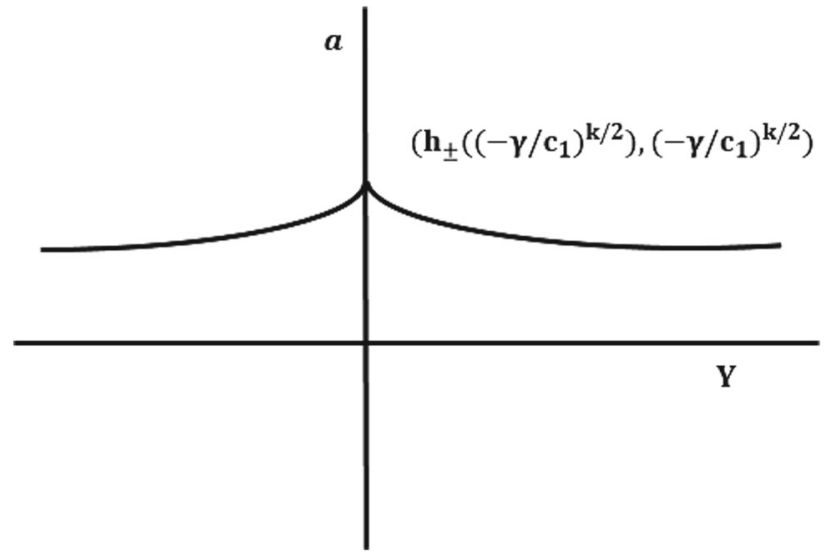

Fig. 5 Graph of the warp factor as a function of $Y$, for $\lambda=(2 k+$ 1)/(2k)

$$
\begin{aligned}
&+\left.(-\gamma)^{k}\left(\ln \left(\frac{-\gamma}{c_{1}}\right)^{k / 2}-\ln a\right)\right), \\
& 0<a \leq\left(\frac{-\gamma}{c_{1}}\right)^{k / 2}
\end{aligned}
$$

and is depicted in Fig. 5.

Again $\rho$ is well defined and continuous at the location of the brane and is equal to

$\rho\left(\left(\frac{-\gamma}{c_{1}}\right)^{k / 2}\right) \equiv \rho(0)=\frac{1}{(-2 \gamma)^{2 k}}$.

Also, the junction condition for the jump of $a^{\prime}$, is identical with the case of the previous section and it is therefore described by Eq. (5.14), while the form of the brane tension that follows from $(5.14)$ is now

$f(\rho(0))=-\frac{\sqrt{6 \rho(0)}}{\kappa_{5}}=-\frac{\sqrt{6}}{\kappa_{5}(-2 \gamma)^{k}}$.

We repeat the procedure for all other values of $1<\lambda<$ $3 / 2$ that lie on the intervals $((n+1) / n,(n-1) /(n-2))$. The two solutions described by Eq. (4.8) are

$$
\begin{aligned}
Y^{+}= & h_{+}(a) \\
= & \frac{\sqrt{6}}{\kappa_{5}}\left(\sum_{s=0}^{n / 2-1} \frac{(-\gamma)^{s}}{2(1-2 s(\lambda-1))}\left(c_{1}^{+} a^{4(\lambda-1)}-\gamma\right)^{1 /(2(\lambda-1))-s}\right. \\
& +\frac{(-\gamma)^{n / 2}\left(c_{1}^{+}\right)^{((n+1)-n \lambda) /(2(\lambda-1))}}{2((n+1)-n \lambda)} a^{2((n+1)-n \lambda)} \\
& \times{ }_{2} F_{1}\left(\frac{(n+1)-n \lambda}{2(1-\lambda)}, \frac{(n+1)-n \lambda}{2(1-\lambda)}, \frac{(n+1)-n \lambda}{2(1-\lambda)}\right. \\
& \left.\left.+1 ; \frac{\gamma}{c_{1}^{+}} a^{4(1-\lambda)}\right)\right)+C_{2}^{+}
\end{aligned}
$$

and

$$
\begin{aligned}
Y^{-}= & h_{-}(a) \\
= & -\frac{\sqrt{6}}{\kappa_{5}}\left(\sum_{s=0}^{n / 2-1} \frac{(-\gamma)^{s}}{2(1-2 s(\lambda-1))}\left(c_{1}^{-} a^{4(\lambda-1)}-\gamma\right)^{1 /(2(\lambda-1))-s}\right. \\
& +\frac{(-\gamma)^{n / 2}\left(c_{1}^{-}\right)^{((n+1)-n \lambda) /(2(\lambda-1))}}{2((n+1)-n \lambda)} a^{2((n+1)-n \lambda)} \\
& \times{ }_{2} F_{1}\left(\frac{(n+1)-n \lambda}{2(1-\lambda)}, \frac{(n+1)-n \lambda}{2(1-\lambda)}, \frac{(n+1)-n \lambda}{2(1-\lambda)}\right. \\
& \left.\left.+1 ; \frac{\gamma}{c_{1}^{-}} a^{4(1-\lambda)}\right)\right)+C_{2}^{-} .
\end{aligned}
$$

By differentiating Eq. (6.13) with respect to $a$, we arrive simply at the integrand function on the left-hand side of Eq. (3.5). After that, finding $h_{+}^{\prime \prime}$ is straightforward and the first two derivatives of $h_{+}$read

$$
\begin{aligned}
h_{+}^{\prime}(a)= & \frac{\sqrt{6}}{\kappa_{5}} \frac{a}{\left(c_{1}^{+}-\gamma a^{4(1-\lambda)}\right)^{1 / 2(1-\lambda)}} \\
= & \frac{\sqrt{6}}{\kappa_{5}}\left(c_{1}^{+} a^{2(\lambda-1)}-\gamma a^{2(1-\lambda)}\right)^{1 /(2(\lambda-1))} \\
h_{+}^{\prime \prime}(a)= & \frac{\sqrt{6}}{\kappa_{5}} a^{2 \lambda-3}\left(c_{1}^{+}+\gamma a^{4(1-\lambda)}\right) \\
& \times\left(c_{1}^{+} a^{2(\lambda-1)}-\gamma a^{2(1-\lambda)}\right)^{(3-2 \lambda) /(2(\lambda-1))} .
\end{aligned}
$$

For our choice of parameters, $h_{+}^{\prime}$ is always positive and $h_{+}^{\prime \prime}$ changes from negative on $\left(0,\left(-\gamma / c_{1}^{+}\right)^{1 /(4(\lambda-1))}\right)$ to positive on $\left(\left(-\gamma / c_{1}^{+}\right)^{1 /(4(\lambda-1))}, \infty\right)$, thus the point

$\left(\left(-\gamma / c_{1}^{+}\right)^{1 /(4(\lambda-1))}, h_{+}\left(\left(-\gamma / c_{1}^{+}\right)^{1 /(4(\lambda-1))}\right)\right)$

is an inflection point of the graph of $h_{+}$. As before, the behavior of the first two derivatives of $h_{-}$can be obtained from those of $h_{+}$since they are opposites.

Joining the two solutions of Eqs. (6.13)-(6.14) at their common inflection point implies

$c_{1}^{+}=c_{1}^{-}=c_{1}$

and

$h_{+}\left(\left(\frac{-\gamma}{c_{1}}\right)^{1 /(4(\lambda-1))}\right)=h_{-}\left(\left(\frac{-\gamma}{c_{1}}\right)^{1 /(4(\lambda-1))}\right)$

and thus provides the following relation between the two constants $C_{2}^{ \pm}$

$$
\begin{aligned}
C_{2}^{+}= & -\frac{\sqrt{6}}{\kappa_{5}}(-\gamma)^{1 /(2(\lambda-1))}\left(\sum_{s=0}^{n / 2-1} \frac{2^{1 /(2(\lambda-1))-s}}{1-2 s(\lambda-1)}+\frac{1}{(n+1)-n \lambda}\right. \\
& \left.\times{ }_{2} F_{1}\left(\frac{(n+1)-n \lambda}{2(1-\lambda)}, \frac{(n+1)-n \lambda}{2(1-\lambda)}, \frac{(n+1)-n \lambda}{2(1-\lambda)}+1 ;-1\right)\right)+C_{2}^{-} .
\end{aligned}
$$

The axis of symmetry is located at $Y=Y_{s}$, with $Y_{s}$ being equal to

$\left.Y_{s}=h_{ \pm}\left(\left(-\gamma / c_{1}\right)^{1 /(4(\lambda-1))}\right)\right)$ 


$$
\begin{aligned}
= & \frac{\sqrt{6}}{2 \kappa_{5}}(-\gamma)^{1 /(2(\lambda-1))}\left(\sum_{s=0}^{n / 2-1} \frac{2^{1 /(2(\lambda-1))-s}}{1-2 s(\lambda-1)}\right. \\
& +\frac{1}{(n+1)-n \lambda} \times{ }_{2} F_{1}\left(\frac{(n+1)-n \lambda}{2(1-\lambda)},\right. \\
& \left.\left.\frac{(n+1)-n \lambda}{2(1-\lambda)}, \frac{(n+1)-n \lambda}{2(1-\lambda)}+1 ;-1\right)\right) \\
& +C_{2}^{+}=-\frac{\sqrt{6}}{2 \kappa_{5}}(-\gamma)^{1 /(2(\lambda-1))}\left(\sum_{s=0}^{n / 2-1} \frac{2^{1 /(2(\lambda-1))-s}}{1-2 s(\lambda-1)}\right. \\
& +\frac{1}{((n+1)-n \lambda)} \\
& \times{ }_{2} F_{1}\left(\frac{(n+1)-n \lambda}{2(1-\lambda)}, \frac{(n+1)-n \lambda}{2(1-\lambda)},\right. \\
& \left.\left.\frac{(n+1)-n \lambda}{2(1-\lambda)}+1 ;-1\right)\right)+C_{2}^{-},
\end{aligned}
$$

and labeling the position of the brane. Positioning the brane at $Y=0$, translates to choosing $Y_{s}=0$ which determines the values of the constants $C_{2}^{ \pm}$in the following way

$$
\begin{aligned}
C_{2}^{+}= & -\frac{\sqrt{6}}{2 \kappa_{5}}(-\gamma)^{1 /(2(\lambda-1))}\left(\sum_{s=0}^{n / 2-1} \frac{2^{1 /(2(\lambda-1))-s}}{1-2 s(\lambda-1)}\right. \\
& +\frac{1}{(n+1)-n \lambda} \\
& \times{ }_{2} F_{1}\left(\frac{(n+1)-n \lambda}{2(1-\lambda)}, \frac{(n+1)-n \lambda}{2(1-\lambda)},\right. \\
& \left.\left.\frac{(n+1)-n \lambda}{2(1-\lambda)}+1 ;-1\right)\right)=-C_{2}^{-} .
\end{aligned}
$$

The matching solution that satisfies the above boundary condition is given by,

$$
\begin{aligned}
|Y|= & \frac{\sqrt{6}}{2 \kappa_{5}}\left([\operatorname{sum}]-\frac{(-\gamma)^{n / 2}\left(c_{1}\right)^{((n+1)-n \lambda) /(2(\lambda-1))}}{(n+1)-n \lambda}\right. \\
& \times a_{2}^{2((n+1)-n \lambda)} F_{1}\left(\frac{(n+1)-n \lambda}{2(1-\lambda)}, \frac{(n+1)-n \lambda}{2(1-\lambda)},\right. \\
& \left.\frac{(n+1)-n \lambda}{2(1-\lambda)}+1 ; \frac{\gamma}{c_{1}} a^{4(1-\lambda)}\right) \\
& +\frac{(-\gamma)^{1 /(2(\lambda-1))}}{(n+1)-n \lambda}{ }_{2} F_{1}\left(\frac{(n+1)-n \lambda}{2(1-\lambda)}\right. \\
& \left.\left.\frac{(n+1)-n \lambda}{2(1-\lambda)}, \frac{(n+1)-n \lambda}{2(1-\lambda)}+1 ;-1\right)\right)
\end{aligned}
$$

where

$0<a \leq\left(\frac{-\gamma}{c_{1}}\right)^{1 / 4(\lambda-1)}$

and we have set the expression [sum] to be equal to,

$$
\sum_{s=0}^{n / 2-1} \frac{1}{1-2 s(\lambda-1)}\left((-\gamma)^{1 /(2(\lambda-1))} 2^{1 /(2(\lambda-1))-s}\right.
$$

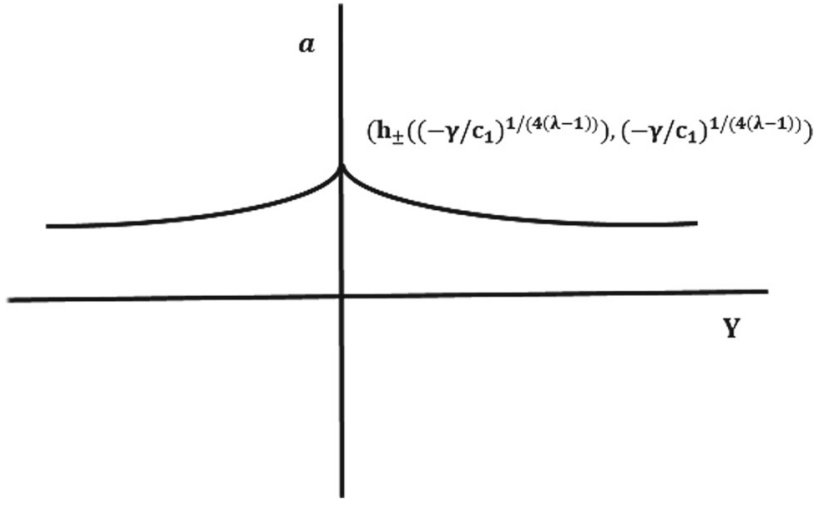

Fig. 6 Graph of the warp factor as a function of $Y$, for $(n+1) / n<$ $\lambda<(n-1) /(n-2)$

$$
\left.-(-\gamma)^{s}\left(c_{1} a^{4(\lambda-1)}-\gamma\right)^{1 /(2(\lambda-1))-s}\right) .
$$

This matching solution is then depicted in Fig. 6.

The value of $\rho$ there is equal to

$\rho\left(\left(\frac{-\gamma}{c_{1}}\right)^{1 /(4(\lambda-1))}\right) \equiv \rho(0)=(-2 \gamma)^{1 /(1-\lambda)}$.

Once more, the junction condition for the jump of $a^{\prime}$, is given by Eq. (5.14), whereas the brane tension becomes now

$f(\rho(0))=-\frac{\sqrt{6 \rho(0)}}{\kappa_{5}}=-\frac{\sqrt{6}}{\kappa_{5}}(-2 \gamma)^{1 /(2(1-\lambda))}$.

Finally, for $\lambda>3 / 2$, the construction of a matching solution follows along the same steps since the solution of Eq. (4.2) can be obtained from Eq. (4.8) by inputting $n=2$.

\section{Localization}

We are going to calculate the Planck mass for the matching solutions we constructed in the previous section, for $\lambda>1$.

First, we derive the behaviour of $a^{2}$ as $Y \rightarrow-\infty$ from Eq. (6.10), we find

$a^{2} \sim e^{\sqrt{6} \kappa_{5} Y(-\gamma)^{-k} / 3}$.

We can write the integral of Eq. (5.16) in the following form

$$
\begin{aligned}
\int_{-Y_{c}}^{Y_{c}} a^{2} d Y & =2 \int_{-Y_{c}}^{0} a^{2} d Y \sim 2 \int_{-Y_{c}}^{0} e^{\sqrt{6} \kappa_{5} Y(-\gamma)^{-k} / 3} d Y= \\
& =\frac{\sqrt{6}}{\kappa_{5}}(-\gamma)^{k}\left(1-e^{-\sqrt{6} \kappa_{5} Y_{c}(-\gamma)^{-k} / 3}\right) .
\end{aligned}
$$

Taking $Y_{c} \rightarrow \infty$ and since $\gamma<0$, we find that the Planck mass remains finite and is proportional to the value

$$
\frac{\sqrt{6}}{\kappa_{5}}(-\gamma)^{k} \text {. }
$$


Finally, we deduce the behaviour of $a^{2}$ as $Y \rightarrow-\infty$ by transforming Eq. (6.22) according to Eq. (4.10), we obtain

$a^{2} \sim\left(-\frac{c_{1}}{\gamma}\right)^{1 /(2(1-\lambda))} e^{\kappa_{5}((n+1)-n \lambda)(-\gamma)^{1 /(2(1-\lambda))} Y /(\sqrt{6}(1-\lambda))}$.

The integral of Eq. (5.16) can then be written as

$$
\begin{aligned}
\int_{-Y_{c}}^{Y_{c}} a^{2} d Y= & 2 \int_{-Y_{c}}^{0} a^{2} d Y \sim 2 \int_{-Y_{c}}^{0}\left(-\frac{c_{1}}{\gamma}\right)^{1 /(2(1-\lambda))} \\
& \times e^{\kappa_{5}((n+1)-n \lambda)(-\gamma)^{1 /(2(1-\lambda))} Y /(\sqrt{6}(1-\lambda))} d Y \\
= & \frac{2 \sqrt{6}(-\gamma)^{1 /(2(\lambda-1))}(1-\lambda)}{\kappa_{5}((n+1)-n \lambda)}\left(-\frac{c_{1}}{\gamma}\right)^{1 /(2(1-\lambda))} \\
& \times\left(1-e^{-\kappa_{5}((n+1)-n \lambda)(-\gamma)^{1 /(2(1-\lambda))} Y_{c} /(\sqrt{6}(1-\lambda))}\right) .
\end{aligned}
$$

As $Y_{c} \rightarrow \infty$ and since $\gamma<0$ and $((n+1)-n \lambda) /(1-\lambda)>0$, the Planck mass remains finite and is proportional to the value

$\frac{2 \sqrt{6}(-\gamma)^{1 /(\lambda-1)}(1-\lambda) c_{1}^{1 /(2(1-\lambda))}}{\kappa_{5}((n+1)-n \lambda)}$.

We can check the behavior of the Planck mass for $(n+$ $1) / n<\lambda<(n-1) /(n-2)$ and as $\lambda$ approaches one. We can choose $\lambda=1+1 /(n-1)$, then clearly $\lambda$ lies on the interval $((n+1) / n,(n-1) /(n-2))$ and approaches one from the right as $n \rightarrow \infty$. Inputting this value in (7.6), we find

$\frac{2 \sqrt{6}}{\kappa_{5}}\left(-\frac{\gamma}{\sqrt{c_{1}}}\right)^{n-1}$

from which we see that for large values of $n$, the behavior of the Planck mass depends on the ordering between $-\gamma$ and $c_{1}$ :

$$
\left(-\frac{\gamma}{\sqrt{c_{1}}}\right)^{n-1} \rightarrow\left\{\begin{aligned}
0,-\gamma & <\sqrt{c_{1}} \\
1,-\gamma & =\sqrt{c_{1}} \\
\infty,-\gamma & >\sqrt{c_{1}}
\end{aligned}\right.
$$

Summarizing, all solutions for $\lambda>1$, are free from finitedistance singularities, they are compatible with the strong and null energy conditions and at the same time lead to a finite Planck mass without imposing any further conditions on the parameters of our model. All solutions for a linear equation of state $(\lambda=1)$ on the other hand, suffered from finite-distance singularities that could only be avoided by a matching mechanism [4]. Still as we showed in [4], for the regular matching solutions, the requirement of a finite Planck mass, leads to a further restriction on $\gamma$, namely that of $-2<\gamma<-1$, which turns out to be incompatible with the requirements of the energy conditions that confine $\gamma$ to values at least greater than -1 . We therefore see that the effect of the non-linear equation of state is significant in the sense that it rectifies previous findings that were based on a linear equation of state.

\section{Conclusions}

In this paper, we studied the behaviour of solutions of a $5 \mathrm{~d}$ brane-world equations in the bulk, with particular emphasis on their regularity properties and associated possibility of localisation of gravity on a flat brane. We showed that the behaviour of all solutions depends critically on the non-linear equation of state parameter $\lambda\left(p=\gamma \rho^{\lambda}\right)$. We showed that solutions with $\lambda<1$ cannot be regular and at the same time localise gravity on the brane. However, the main result of this work is that when $\lambda>1$, there are solutions which are free of singularities and having finite Planck mass, that is they are able to localise gravity on the brane. We also showed that the behaviour of the matched solutions is consistent in the sense that they are convergent in the correct intervals for their matching to be allowed. It is an interesting open question whether such non-linear equation of state satisfying the null energy condition can be realised using an underlying microscopic description.

Acknowledgements Work partially performed by I.A. as International professor of the Francqui Foundation, Belgium.

Data Availability Statement This manuscript has no associated data or the data will not be deposited. [Authors' comment: This work does not use any data.]

Open Access This article is licensed under a Creative Commons Attribution 4.0 International License, which permits use, sharing, adaptation, distribution and reproduction in any medium or format, as long as you give appropriate credit to the original author(s) and the source, provide a link to the Creative Commons licence, and indicate if changes were made. The images or other third party material in this article are included in the article's Creative Commons licence, unless indicated otherwise in a credit line to the material. If material is not included in the article's Creative Commons licence and your intended use is not permitted by statutory regulation or exceeds the permitted use, you will need to obtain permission directly from the copyright holder. To view a copy of this licence, visit http://creativecomm ons.org/licenses/by/4.0/.

Funded by $\mathrm{SCOAP}^{3}$.

\section{References}

1. I. Antoniadis, S. Cotsakis, I. Klaoudatou, Braneworld cosmological singularities, Proceedings of MG11 meeting on General Relativity, vol. 3, pp. 2054-2056. arXiv:0701033 [gr-qc]

2. I. Antoniadis, S. Cotsakis, I. Klaoudatou, Brane singularities and their avoidance. Class. Quantum Gravity 27, 235018 (2010). arXiv: 1010.6175 [gr-qc]

3. I. Antoniadis, S. Cotsakis, I. Klaoudatou, Brane singularities with mixtures in the bulk. Fortschr. Phys. 61, 20-49 (2013). arXiv: 1206.0090 [hep-th]

4. I. Antoniadis, S. Cotsakis, I. Klaoudatou, Enveloping branes and braneworld singularities. Eur. Phys. J. C 74, 3192 (2014). arXiv:1406.0611v2 [hep-th]

5. I. Antoniadis, S. Cotsakis, I. Klaoudatou, Curved branes with regular support. Eur. Phys. J. C 76, 511 (2016). arXiv:1606.09453 [hep-th] 
6. N. Arkani-Hamed, S. Dimopoulos, N. Kaloper, R. Sundrum, A small cosmological constant from a large extra dimension. Phys. Lett. B 480, 193-199 (2000). arXiv:0001197v2 [hepth]

7. S. Kachru, M. Schulz, E. Silverstein, Bounds on curved domain walls in 5d gravity. Phys. Rev. D 62, 085003 (2000). arXiv:0002121 [hep-th]

8. S.S. Gubser, Curvature singularities: the good, the bad, and the naked. Adv. Theor. Math. Phys. 4, 679-745 (2000). arXiv:0002160 [hep-th]

9. S.K. Srivastava, Future universe with $w<-1$ without big smash. Phys. Lett. B 619, 1-4 (2005). arXiv:0407048v4 [astro-ph]

10. P.F. Gonzalez-Diaz, You need not be afraid of phantom energy. Phys. Rev. D 68, 021303 (2003). arXiv:0305559 [astro-ph]

11. A.V. Astashenok, S. Nojiri, S.D. Odintsov, A.V. Yurov, Phantom cosmology without big rip singularity. Phys. Lett. B 709, 396-403 (2012). arXiv:1201.4056 [gr-qc]

12. J.D. Barrow, Graduated inflationary universes. Phys. Lett. B 235, 40-43 (1990)

13. A.Y. Kamenshchik, U. Moschella, V. Pasquier, An alternative to quintessence. Phys. Lett. B 511, 265-268 (2001). arXiv:0103004 [gr-qc]

14. M.C. Bento, O. Bertolami, A.A. Sen, Generalized Chaplygin gas, accelerated expansion and dark energy-matter unification. Phys. Rev. D 66, 043507 (2002). arXiv:0202064 [gr-qc]
15. S. Cotsakis, I. Klaoudatou, Future singularities of isotropic cosmologies. J. Geom. Phys. 55, 306-315 (2005). arXiv:0409022 [grqc]

16. S. Cotsakis, I. Klaoudatou, Cosmological singularities and Bel-Robinson energy. J. Geom. Phys. 57, 1303-1312 (2007). arXiv:0604029 [gr-qc]

17. S. Nojiri, S.D. Odintsov, S. Tsujikawa, Properties of singularities in (phantom) dark energy universe. Phys. Rev. D 71, 063004 (2005). arXiv:0501025 [hep-th]

18. R.M. Wald, General Relativity (University of Chicago Press, Chicago, 1984)

19. E. Poisson, A Relativist's Toolkit (Cambridge University Press, 2004)

20. I. Antoniadis, S. Cotsakis, I. Klaoudatou, (in preparation)

21. A. Erdelyi (ed.), Bateman Manuscript Project, Higher Transcendental Functions, vol. 1 (McGraw-Hill Book Company, 1953)

22. P.M. Morse, H. Feshbach, Methods of Theoretical Physics, vol. 1, Sect. 4.3 (McGraw-Hill, 1953)

23. S. Forste, H.P. Nilles, I. Zavala, Nontrivial cosmological constant in Brane worlds with unorthodox Lagrangians. JCAP 07, 007 (2011). arXiv:1104.2570 [hep-th] 